\title{
Research on the power signal processing system space discharge location
}

\author{
Yutong Chen, Tianzheng Wang, Hua Yu, Yanpeng Li \\ State Grid Shanxi Electric Power Research Institute, Shanxi 030001, China
}

Keywords: Power processing, signal discharge, positioning research.

\begin{abstract}
Stable operation of the power system is inseparable from the normal operation of a variety of electrical equipment; partial discharge signal power processing system to determine the time difference is the basis of the PD source localization. Typical methods of feature points difference estimate the time difference, the difference based on generalized correlation time, seek time difference based on the frequency domain, the parametric model law. In this paper, starting from two aspects, one to study the application of the theory of Hilbert - Huang transform transformer partial discharge signal spectrum and marginal spectrum analysis, and has done a simulation experiment results show that the application of the transformer partial discharge fault type the recognition is very effective; on the other hand, a partial discharge of the design expert system based on fuzzy reasoning transformer, this system is built in the traditional expert systems and fuzzy inference and fuzzy reasoning is based on the an intelligent decision-making method based on fuzzy mathematics, has been widely used in various fields, intelligent fault diagnosis of this article will be applied to the transformer partial discharge. Finally, a simulation experiment, this paper for transformer fault diagnosis, especially transformer partial discharge pattern recognition has a very important reference value.
\end{abstract}

\section{Introduction}

Stable operation of the power system is inseparable from the proper functioning of a variety of electrical equipment, power systems and power transformer is the most important high-voltage electrical equipment, which bears the important task of the current and voltage conversion and power transmission, power transformers to run properly not directly affect the operation of the power system security and stability, so we vividly seen as grid power transformer "hub." The most widely used is the two methods of partial discharge pattern recognition methods currently in use: one is called time-domain analysis, there is a statistical analysis, with the progress of modern science and technology as well as storage fast sampling technology, making local discharge pulse pattern recognition have been developed, more scientific and intelligent pattern recognition. Transformer partial discharge monitoring originated in the 20th century, when the researchers only partial discharge pulse waveform discharge qualitative analysis. About power transformer fault diagnosis, especially for transformer partial discharge detection problem, many researchers partial discharge detection equipment related to the development, PD signal detection equipment has become increasingly diverse, for partial discharge location of the power transformer technology becomes more mature, of course, this maturing transformer PD online monitoring technology for rapid troubleshooting and transformer protection and stable operation of the power system is of great significance. In this paper, starting from two aspects, one to study the application of the theory of Hilbert - Huang change the transformer partial discharge signals HHT marginal spectrum and spectrum analysis, and has done a simulation. On the other hand the design of the transformer partial discharge expert system based on fuzzy reasoning, and this system was simulated. Experimental results show that the fuzzy reasoning applied to transformer partial discharge intelligent fault diagnosis is effective. 


\section{Signal Processing System Analysis}

When the natural frequency of the same signal characteristics similar, the distribution of the wavelet transform with basic functions also when the scale decreases with increasing frequency, a signal suitable for analysis of an arbitrary scale. Because the wavelet packet transform high frequency components of the signal can be further broken down, the signal can be decomposed into the same bandwidth as many bands, but they contain the number of harmonics are also the same. Therefore, wavelet packet analysis, compared with the wavelet analysis, it has wider application ${ }^{[1-2]}$. In the multi-resolution analysis, given the orthogonal wave function $\Psi(\mathrm{t})$ and the scaling function $\varphi(t)$, the other scales relationship:

$$
\begin{aligned}
& \Phi(\mathrm{t})=\sqrt{2} \sum_{k \in z} h(k) \Phi(2 t-k) \\
& \psi(\mathrm{t})=\sqrt{2} \sum_{k \in z} g(k) \Phi(2 t-k)
\end{aligned}
$$

Construction sequence by the formula (1) and (2) $\left\{\Phi_{n}(\mathrm{t})\right\}$,) among them $n \in \mathrm{z}^{+} \psi$ ) Called orthogonal wavelet packet determined by wavelet. Wavelet packet space can be further decomposed, thereby increasing the frequency resolution. Wavelet packet reconstruction algorithm is as follows:

$$
d_{l}^{j+1}=\sum_{\mathrm{k}}\left(h_{1-2} d_{k}^{j, 2 n}+g_{1-2 k} d_{k}^{j, 2 n+1}\right)
$$

In comparison with the wavelet transform, wavelet packet transform even the high frequency part of the signal can be further broken down, with more versatility. Wavelet transform compared to Fourier transform, it has the advantage in that the time domain and frequency domain localized transform, wavelet transform more efficiently extract feature information signal, and also through the translation function stretching operations, etc., a function of pretreatment or pre-processed signal for further refinement of the multi-scale decomposition, thus realizing the Fourier transform cannot be achieved in a number of functions.

\section{The digital signal analysis and processing of partial discharge analysis}

Since the inside of the transformer or the surface of the insulator, the insulator inside appeared uneven field strength, these uneven front may cause localized discharge phenomenon occurs in certain areas. Under the influence of the electric field at the edge or the air gap insulated conductors within the structure of the transformer, the occurrence of non-penetrating film discharge phenomenon, we called partial discharge NEHuang Chinese American scientists put forward a nonlinear analysis in 1998, A new method of non-stationary signals (Hilbert - Huang Transform). Experimental results show that the proposed treated by partial discharge signal in favor of partial discharge type identification, partial discharge pattern recognition and insulation defect diagnosis is important ${ }^{[3-4]}$. Many scholars in the analysis of partial discharge model of power transformers with three capacitor model, the paper also cited the most common three-capacitance model, analyze partial discharge transformer theory.

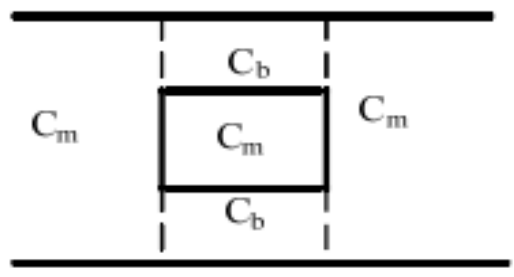

(a) a sectional bubble-containing medium

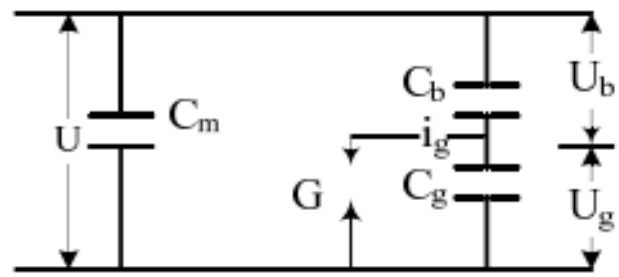

(b) a sectional bubble-containing medium

Figure 2 three capacitance model

Many scholars in the analysis of partial discharge model of power transformers with three capacitor model, the paper also cited the most common three-capacitance model, analyze partial 
discharge transformer theory. Partial discharge of the emergence and development depends not only on the media type, the electrode structure, but also with electrical insulation defects form are inseparable, so the partial discharge pulse current (including amplitude, wave, etc.) will also be affected by these factors.

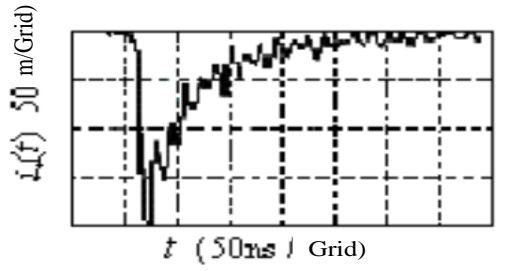

(a) Oil cardboard insulation gap discharge pulse

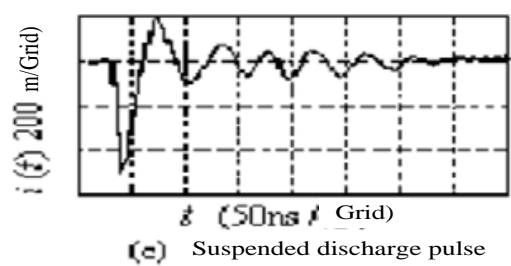

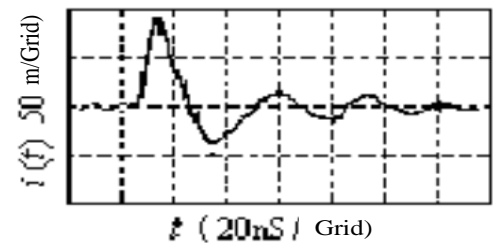

(b) Oil gap discharge positive pulse

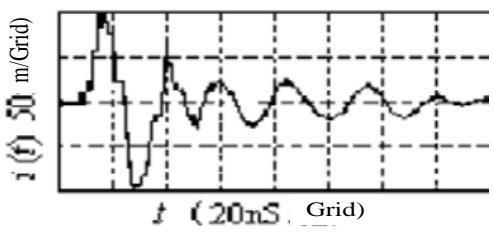

(d) Surface discharge pulse

Figure 3 partial discharge pulses

From Figure 3, we can see, the typical partial discharge pulse waveform has spark, corona, intermittent discharge breakdown of several stages; oil discharges and solid formation of bubbles forming gap discharge pulse waveform closer; is very similar to the oil discharge occurs in the air gap of the waveform; suspended discharge is not in the form of a separate discharge, from the point of view of its waveform, the overall performance of other types of discharge.

\section{Signal Processing System Analysis}

In the discharge amount put on the access to local television. Depending on the amount of discharge can directly reflect the insulation transformer partial discharge status, but also the state of partial discharge inside the transformer important basis for the evaluation of partial discharges. In addition, the switching operation of the power system and the corona signals generated by transient pulse signal is transformer partial discharge the most important interference detection characteristics of these interfering signals and partial discharge signals and similar, which provides for on-site detection of a problem ${ }^{[5-6]}$. Figure shows the schematic diagram of the pulse current sensor 4 . Pulse current sensor applications ferrite core around the system, the bandwidth of the sensor is about $4 \mathrm{KHZ}$ $\sim 1.2 \mathrm{MHZ}$, an active broadband type. Preamplifier 1 or 10 times magnification selection mode is manual.

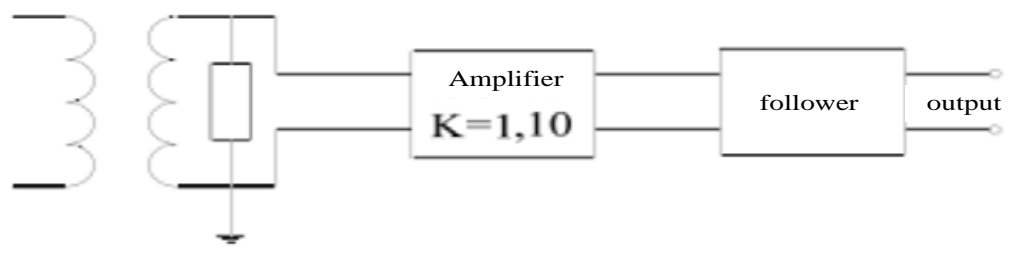

Figure 4 Schematic diagram of the pulse current sensor

Figure 5 shows the attenuator and amplifier unit schematic. Attenuator consisting of a network of resistance and capacitance in order to obtain the best frequency characteristics. Attenuator multiples are 1, 1 / 2, 1 / 4, 1 / 8. Contains two amplifiers, each amplifier magnification 1, 2, 4, 8 respectively. Both attenuation and amplification of multiple process control can be achieved.

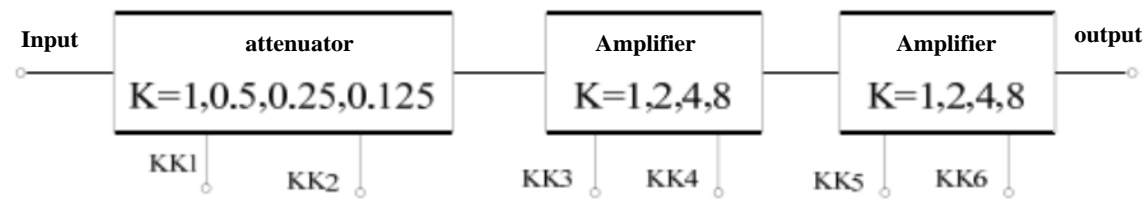


Figure 5 attenuator and amplifier unit schematics

$3 \mathrm{~dB}$ bandwidth of the amplifier has 10KHZ 2MHZ. In order to obtain good frequency characteristics, selection miniature electrical control attenuator, amplifier range switch, Fig. 5 the relay control signal generated by the decoded address ${ }^{[7-8]}$. Have been finalized for the fuzzy inference system, fuzzy implication relationship is finalized, but the synthesis of the calculation method is not unique. Depending on the method of synthesis of computing, fuzzy inference methods can be divided into different categories.

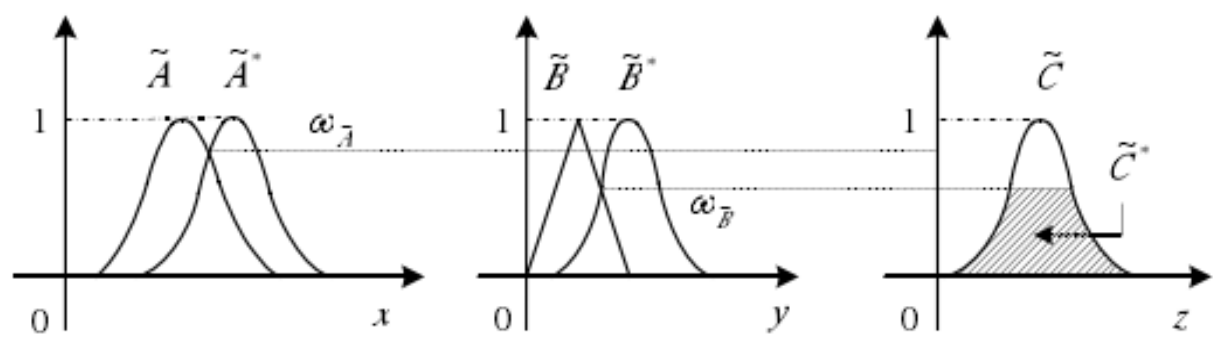

Figure 6 Single premise Mamdani fuzzy reasoning rules

Since part of the fuzzy rules prerequisites by the conjunction "and" connected together, so called excitation intensity or degree of satisfaction of fuzzy rules, which indicates the degree of the antecedent part of the rule is satisfied. Figure 6 shows a single rule more than the antecedent of Madani fuzzy reasoning, this conclusion can be extended directly to the case with more than two former members. Concluded: For multiple rules under multiple preconditions fuzzy reasoning, fuzzy relations under each fuzzy rules and apply the appropriate set of multiple rules for processing.

\section{Summary}

Electrical power systems by detecting space discharge signals; you can quickly and accurately locate the position of the fault in the power equipment failure to timely replacement of faulty equipment, to ensure the normal operation of the power system. To achieve PD locate the source, whether it is the use of ultrasonic positioning method or UHF positioning method, the process must involve the delay is the time difference between two or more signals. Therefore, power transformer partial discharge on-line monitoring techniques, to assess the state of transformer partial discharge, achieving a transformer partial discharge fault diagnosis and localization of partial discharge point, timely troubleshooting, preventive measures have also been slowly more people are concerned .

\section{References}

[1] P. Werle, H. Borsi. A New Method for Partial Discharge Location on Power Transformer Based on a System Theoretical Approach. IEEE Tran. On Dielec and Elec. Insul. 2012.

[2] Wang Z D, Crossley P A, Cornick K J, et al. Partial Discharge Locationin Power Transformers. IEE Proc. Sci. Meas. Technol., 2011.

[3]M. Hawkes, A. Nehorai. Wideband Source Localization Using a Distributed Acoustic Vector-Sensor Array [J]. IEEE Trans. on Signal Processing, 2011.

[4] Tabrikian, R. Shavit, D. Rahamim. An Efficient Vector Sensor Configuration for Source Localization. IEEE Signal Procession Letters.2010

[5] Huang N E, Shen Z, Long S R, et al. A new view of nonlinear water waves: the Hilbert spectrum. Ann Rev Fluid Mech, 2011.

[6] James R E, Phung B T, Su Q. Application of Digital Filtering Techniques to the Determination of Partial Discharge Location in Transformer 2012.

[7] K. T. Wong. Uni-vector-sensor ESPRIT for multi-source azimuth, elevation, and polarization estimation [J] 2011. 
[8] D.Wenzel, H. Borsi, E Gockenbach. Pulse Shaped Noise Reduction and Partial Discharge Localization on Transformers Using the Karhunen-Loeve-Transform. Proceedings of 9' ISH, 2012. 